\title{
Worst-Case Analysis of the X-38 Crew Return Vehicle Flight Control System
}

\author{
Jong-Yeob Shin* and Gary J. Balas ${ }^{\dagger}$ \\ University of Minnesota, Minneapolis, Minnesota 55455 \\ and \\ Andrew K. Packard \\ University of California, Berkeley, Berkeley, California 94704
}

\begin{abstract}
A linear fractional transformation (LFT) model of the linearized equation of the lateral-directional axes of the X-38 crew return vehicle is developed to facilitate the analysis of flight control systems. The LFT model represents uncertainty in nine aerodynamic stability derivatives at a given flight condition with frequency-domain performance specifications. The X-38 LFT model combined with a controller at specific flight conditions is used to determine the aerodynamic coefficients that result in the worst-case performance and gain/phase margin of the closed-loop system. The objective is to verify that a given controller remains stable and achieves desired performance objectives for all predefined aerodynamic variations at select operating conditions along its flight trajectory.
\end{abstract}

\begin{tabular}{|c|c|}
\hline & Nomenclature \\
\hline$b$ & $=$ wing span, $\mathrm{ft}^{2}$ \\
\hline$C l_{i}$ & $=$ roll moment nondimensional stability derivatives \\
\hline$C l_{i \text { c.g. }}$ & $\begin{array}{l}=\text { roll moment nondimensional stability derivatives } \\
\text { at the center of gravity }\end{array}$ \\
\hline$C n_{i}$ & $=$ yaw moment nondimensional stability derivatives \\
\hline$C n_{i \text { c.g. }}$ & $\begin{aligned}= & \text { yaw moment nondimensional stability derivatives } \\
& \text { at the center of gravity }(i=\beta, p, r, d a, d r)\end{aligned}$ \\
\hline$d a$ & $=$ aileron deflection, $\mathrm{rad}$ \\
\hline$d r$ & $=$ rudder deflection, rad \\
\hline $\begin{array}{l}I_{x x}, I_{x z} \\
I_{y y}, I_{z z}\end{array}$ & $=$ inertial moments, $\operatorname{slug} \mathrm{ft}^{2}$ \\
\hline & $=$ roll moment stability derivatives \\
\hline$l, n$ & $=$ roll, yaw moment, $\mathrm{lb} \mathrm{ft}$ \\
\hline$n_{i}$ & $=$ yaw moment stability derivatives \\
\hline$p$ & $=$ roll rate, $\mathrm{rad} \mathrm{s}^{-1}$ \\
\hline$Q$ & $=$ dynamic pressure, $\mathrm{psi}$ \\
\hline$q$ & $=$ pitch rate, $\mathrm{rad} \mathrm{s}^{-1}$ \\
\hline$r$ & yaw rate, $\mathrm{rad} \mathrm{s}^{-1}$ \\
\hline$S$ & reference area, $\mathrm{ft}^{2}$ \\
\hline$V$ & $=$ velocity, $\mathrm{ft} \mathrm{s}^{-1}$ \\
\hline$Y_{i}$ & $=$ side force stability derivatives \\
\hline$\alpha$ & $=$ angle of attack, rad \\
\hline$\beta$ & $=$ sideslip angle, $\mathrm{rad}$ \\
\hline$\phi$ & $=$ roll angle, $\mathrm{rad}$ \\
\hline
\end{tabular}

\section{Introduction}

$\mathbf{T}$ HE X-38 vehicle is a prototype of the emergency crew return vehicle $(\mathrm{CRV})$ for the International Space Station. The CRV will glide from orbit unpowered and use a steerable parafoil parachute for its final descent to landing. ${ }^{\S}$ The X-38 has a full lifting body flight control system (FCS) that allows the vehicle to fly autonomously prior to parafoil deployment. The X-38 FCS differs slightly from a conventional aircraft control system, as it uses differential body flaps and a rudder for lateral-directionalmotion con-

Received 4 November 1999; accepted for publication 26 June 2000. Copyright (C) 2000 by the authors. Published by the American Institute of Aeronautics and Astronautics, Inc., with permission.

${ }^{*}$ Graduate Research Assistant, Department of Aerospace Engineering and Mechanics. Student Member AIAA.

${ }^{\dagger}$ Associate Professor, Department of Aerospace Engineering and Mechanics. Member AIAA.

¥Professor, Department of Mechanical Engineering.

${ }^{\S}$ Data available online at http://www.dfrc.nasa.gov/Projects/X38/ [cited 29 January 2001]. trol and symmetric body flaps for longitudinal motion control. The airfoil is based on the U.S. Air Force's X-24A project, and the electromechanicalactuators(EMAs) come from a previous joint NASA, Air Force, and Navy research and developmentproject. ${ }^{\S}$ Vehicle 132 (V132) was constructed to test the X-38 FCS prior to Vehicle 201, (V201), which will launch this year from the shuttle bay doors.

The X-38 aerodynamic coefficients were measured in wind tunnel tests and are defined by a nominal value with a range of variation. These aerodynamic variations or uncertainties have to be accounted for in the dynamic model of the X-38 for flight control analysis, because the closed-loop system must remain stable in the face of these uncertainties. The aerodynamic coefficients occur as parameters in the dynamic model; hence it is straightforward to model uncertain aerodynamic coefficients as having a nominal value with a range of variations by means of linear fractional transformations (LFTs). ${ }^{1,2}$ For more details on the modeling of parametric uncertainty by LFTs, see Refs. 3-5.

Guaranteeing robustness of the X-38 FCS to the aerodynamic coefficient uncertainties is important for the flight test. The current approach taken by NASA Johnson Space Center to analyze the robustness of the X-38 FCS is to select 128 worst-case aerodynamic coefficient parameter sets to test. For each worst-case set, closedloop gain and phase margins are calculated to determine if the desired specifications are met. An alternative approach to robustness analysis based on probability theory is the stochastic robust analysis proposed by Stengel and Ray. ${ }^{6,7}$ The probability of instability is defined based on the probability distributions of closed-loopeigenvalues, given the statistics of the bounded uncertain parameter. The probability of instability in the confidence level is calculated by using the Monte Carlo method. The stochastic robustness analysis method is dependent on the number of trials and requires intensive computation.

A deterministic approach to robust analysis is based on the structured singular value $(\mu)$ analysis. ${ }^{8}$ An example of $\mu$ analysis for robust analysis is provided in a paper on the robustness of space shuttle lateral-directionalaxis FCS that is due to uncertain aerodynamic coefficients.

Structured singular value $(\mu)$ analysis techniques are used to determine the stability robustness and performance robustness with respect to changes in aerodynamic parameters. Methods to calculate bounds on the structured singular value have been developed, as solving for the exact value of the structured singular value is a non-parametric-hardproblem. ${ }^{10-14}$ The structured singular value is bounded by lower and upper values as spectral radius and largest singular value of an argument plant, which includes errors, noise variations, disturbances, and uncertain blocks. The power algorithm 
is introduced to calculate lower bounds of $\mu$ with real parameter uncertainties. ${ }^{10}$ The gap between lower and upper bounds of $\mu$ may be large for some problems. The branch-and-bound algorithm is used to reduce the gap for real parameter uncertainties. ${ }^{15,16}$

The worst-case performance analysis is a deterministic measure of the worst-case performance $\mathcal{H}_{\infty}$ norm of a linear system for a fixed size of uncertainty. Worst-case performance is directly related to skewed $\mu^{13,17}$ and is the robustness analysis approach taken in this paper to analyze candidate flight controllers for the X-38 V132.

The focus of the paper is on the analysis of the lateral-directional axis flight controller for the X-38 V132. Section II presents the derivation of the LFT model of the X-38 lateral-directionalaxis with the aerodynamic stability derivative uncertainties. In Sec. III, the interconnection of X-38 and properties of coupled uncertainties is presented. The worst-case analysis algorithmis presented in Sec. IV, and the worst-case analysis results of the controlled X-38 system are presented in Sec. V. The worst-case analysis technique is also used to calculate a measure of the minimum combined gain and phase margins for each input/output channel in Sec. VI. Section VII concludes with a summary of the results.

\section{X-38 Lateral-Directional Model}

\section{A. Linearized Equation}

We model the X-38 aircraft as a rigid body to derive equations of a lateral-directionalmotion. Assuming that pitch rate is constant, we can separate rigid-body motion into lateral-directionaland longitudinal axis motion. Equations of motions for the lateral-directional axes can be written as ${ }^{18}$

$$
\begin{aligned}
& I_{x x} \dot{p}-I_{x z} \dot{r}=l+\left(I_{y y} r+I_{x z} p-I_{z z} r\right) q \\
& -I_{x z} \dot{p}+I_{z z} \dot{r}=n+\left(I_{x x} p-I_{x z} r-I_{y y} p\right) q \\
& \dot{\phi}=p+\tan (\theta) r \\
& \dot{\beta}=Y_{b} \beta+\left(w_{0} / V+Y_{p}\right) p+\left(Y_{r}-u_{0} / V\right) r \\
& \quad+Y_{d a} d a+Y_{d r} d r+[g \cos (\gamma) / V] \phi
\end{aligned}
$$

where $g$ is gravity, $w_{0}$ is $V \sin (\alpha), u_{0}$ is $V \cos (\alpha)$, and $\gamma$ is an angle between a horizontal line and a flight velocity vector. The roll moment, $l$, and yaw moment, $n$, can be written as a function of lateral-directional nondimensional derivatives as shown in Eqs. (5) and $(6)^{18}$ :

$$
\begin{aligned}
l= & \frac{Q S b}{I_{x x}}\left(C l_{\beta \text { c.g. }} \beta+\frac{b}{2 V} C l_{p \text { c.g. }} p+\frac{b}{2 V} C l_{r \text { c.g. }} r\right. \\
& \left.+C l_{d a_{\text {c.g. }}} d a+C l_{d r_{\text {c.g. }}} d r\right) \\
n= & \frac{Q S b}{I_{z z}}\left(C n_{\beta \text { c.g. }} \beta+\frac{b}{2 V} C n_{p \text { c.g. }} P+\frac{b}{2 V} C n_{r \text { c.g. }} r\right. \\
& \left.+C n_{d a_{\text {c.g. }}} d a+C n_{d r_{\text {c.g. }}} d r\right)
\end{aligned}
$$

The subscript cg represents the X-38 center of the gravitational point. The nondimensional stability derivatives obtained from a wind tunnel test are measured at the aerodynamic center. ${ }^{19}$ The derivatives at the center of the gravitational point can be derived from the derivatives at the aerodynamic center of X-38, which is not same as the center of the gravitational point. These relationships are given as

$$
\begin{gathered}
C l_{i \text { c.g. }}=C l_{i}-\left(Z_{f} / b\right) C y_{i} \\
C n_{i \text { c.g. }}=C n_{i}+\left(X_{f} / b\right) C y_{i}, \quad i=\beta, p, r, d a, d r
\end{gathered}
$$

where $Z_{f}(\mathrm{ft})$ and $X_{f}(\mathrm{ft})$ are positions of the center of the gravitational point of X-38 from the aerodynamic point. From Eqs. (1) and (2), the change on the roll rates and yaw rates can be written as follows:

$$
\begin{aligned}
\dot{p}= & D_{I}\left\{l+\left(q / I_{x x}\right)\left(I_{y y} r+I_{x z} p-I_{z z} r\right)\right. \\
& \left.+\left(I_{x z} / I_{x x}\right)\left[n+\left(q / I_{z z}\right)\left(I_{x x} p-I_{x z} r-I_{y y} p\right)\right]\right\} \\
\dot{r}= & D_{I}\left\{\left(I_{x z} / I_{z z}\right)\left[l+\left(q / I_{x x}\right)\left(I_{y y} r+I_{x z} p-I_{z z} r\right)\right]\right. \\
& \left.+n+\left(q / I_{z z}\right)\left(I_{x x} p-I_{x z} r-I_{y y} p\right)\right\}
\end{aligned}
$$

where

$$
D_{I}=\left(1-\frac{I_{x z} I_{x z}}{I_{x x} I_{z z}}\right)^{-1}
$$

The state variables are $\beta, p, r$, and $\phi$ and the inputs are the differentialdeflections of the body flaps, $d a$, and the deflection of a rudder, $d r$. The output variables are $\beta, p, r, \phi$ and lateral acceleration, $N_{y}$, defined as

$$
N_{y}=N_{y \text { c.g. }}+x_{a} \dot{r}-z_{a} \dot{p}
$$

where $N_{y \text { c.g. }}$ is a lateral acceleration at the center of the gravitation, and $x_{a}(\mathrm{ft})$ and $z_{a}(\mathrm{ft})$ are the positions of the acceleration sensor. The equations of the linearized lateral-directionalmotion are written out in full detail in the Appendix.

\section{B. X-38 LFT Model}

The X-38 aerodynamic data have uncertainty in the following nondimensional stability derivatives: $C l_{\beta}, C l_{d a}, C l_{d r}, C y_{\beta}, C y_{d a}$, $C y_{d r}, C n_{\beta}, C n_{d a}$, and $C n_{d r}$. Uncertainty in stability derivatives can be described by a nominal nondimensional aerodynamic derivative with a bounded range of possible values. For example, $C l_{\beta}$ is measured as a -0.497 nominal value with \pm 0.100 variation calculated based on five $\sigma$ statistics. ${ }^{19}$ In this case, $C l_{\beta}$ can be described as follows:

$$
C l_{\beta}=C l_{\beta \mathrm{nom}}+\delta_{C l_{\beta}} \mathrm{d} C l_{\beta}
$$

where $\delta_{C l_{\beta}}$ is an uncertainty real parameter whose range is \pm 1 and $\mathrm{d} C l_{\beta}$ represents the magnitude of the uncertain range (0.100). It is straightforward to represent a parameter with a nominal value and an uncertain range as a LFT model with structured uncertainty. ${ }^{20}$ The stability derivative, $C l_{\beta}$, can be represented as a lower LFT model with an uncertainty real parameter, $\delta_{C_{\beta}}$ :

$$
C l_{\beta}=F_{l}\left(M_{C l_{\beta}}, \delta_{C l_{\beta}}\right)
$$

where

$$
M_{C l_{d a}}=\left[\begin{array}{cc}
C l_{\beta \mathrm{nom}} & \mathrm{d} C l_{\beta} \\
1 & 0
\end{array}\right]
$$

and $F_{l}(M, \Delta)$ is defined as

$$
F_{l}(M, \Delta)=\left[M_{11}+M_{12} \Delta\left(I-M_{22} \Delta\right)^{-1} M_{21}\right]
$$

We can describe the nine robust stability derivatives as LFT models with nine uncertain real parameters related with state-space equations. The representation of parametric uncertainty in a state-space equation was presented by Morton and McAfoos. ${ }^{8}$ A more detailed description of uncertainty modeling can be found in Ref. 20.

The lateral-directionalstate-space equations for $\dot{\beta}, \dot{p}$, and $\dot{r}$ with respect to the nine nondimensional stability derivatives are

$$
\left[\begin{array}{c}
\dot{\beta} \\
\dot{p} \\
\dot{r}
\end{array}\right]=\left[\begin{array}{lll}
M_{\beta} V_{\beta} & M_{p} & M_{r}
\end{array}\right]\left[\begin{array}{c}
\beta \\
p \\
r
\end{array}\right]+\left[\begin{array}{ll}
M_{d a} V_{d a} & M_{d r} V_{d r}
\end{array}\right]\left[\begin{array}{c}
d a \\
d r
\end{array}\right]
$$




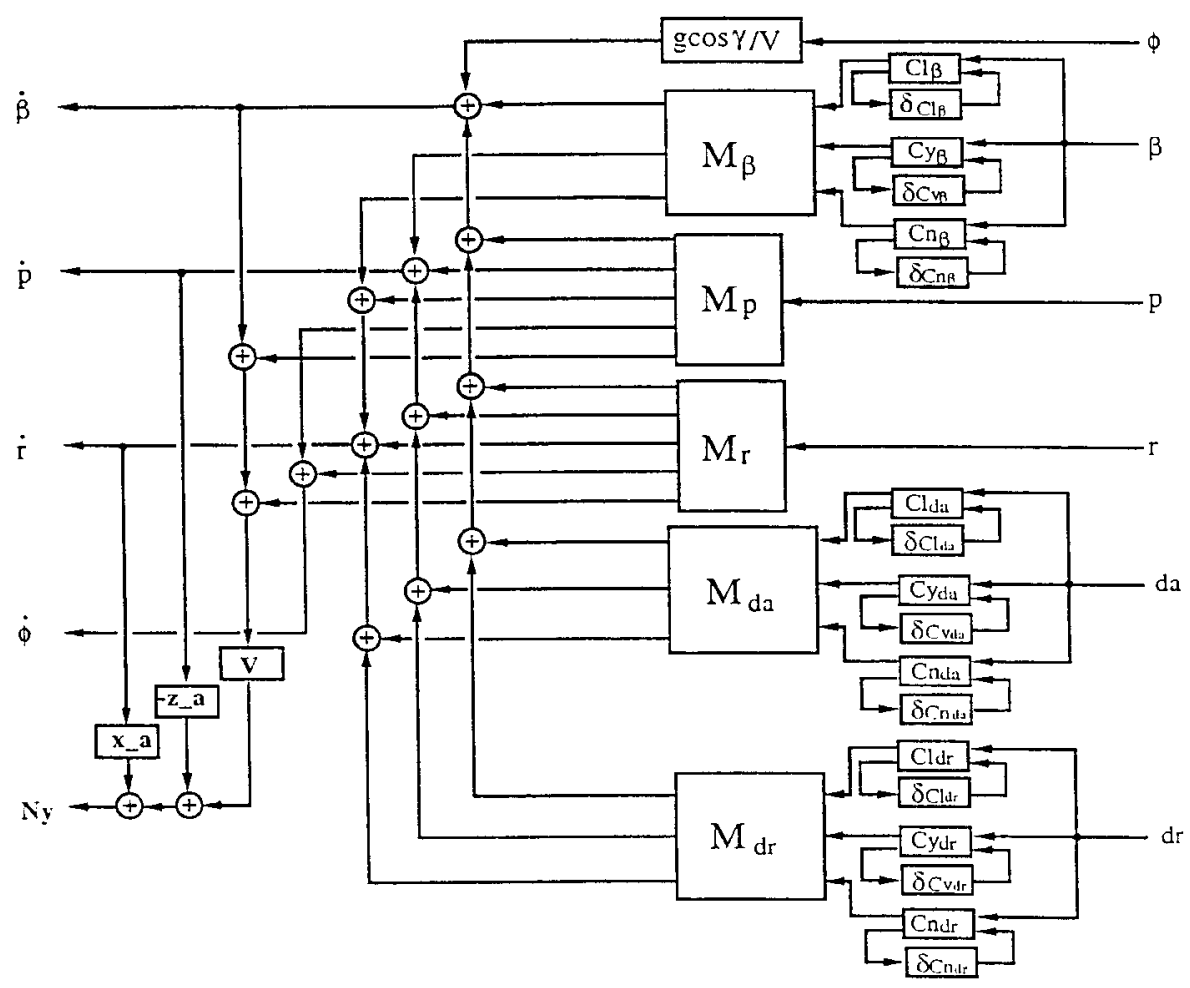

Fig. 1 Block diagram of the lateral-directional state-space equations for the X-38.

where

$$
\begin{aligned}
& M_{\beta}=\left[\begin{array}{ccc}
0 & Q S / \operatorname{mass} V & 0 \\
a_{1} & \left(a_{1} / b\right)\left[\left(I_{x z} / I_{z z}\right) X_{f}-Z_{f}\right] & a_{1}\left(I_{x z} / I_{z z}\right) \\
a_{2} & \left(a_{2} / b\right)\left(X_{f}-I_{x z} / I_{x x}\right) & a_{2}\left(I_{x z} / I_{x x}\right)
\end{array}\right] \\
& M_{p}=\left[\begin{array}{c}
A_{\dot{\beta} p} \\
A_{\dot{p} p} \\
A_{\dot{r} p}
\end{array}\right] \\
& M_{r}=\left[\begin{array}{c}
A_{\dot{\beta} r} \\
A_{\dot{p r r}} \\
A_{\dot{r} r}
\end{array}\right] \\
& M_{d a}=\left[\begin{array}{ccc}
0 & Q S / \operatorname{mass} V & 0 \\
a_{1} & \left(a_{1} / b\right)\left[\left(I_{x z} / I_{z z}\right) X_{f}-Z_{f}\right] & a_{1}\left(I_{x z} / I_{z z}\right) \\
a_{2} & \left(a_{2} / b\right)\left[\left(X_{f}-\left(I_{x z} / I_{x x}\right) Z_{f}\right]\right. & a_{2}\left(I_{x z} / I_{x x}\right)
\end{array}\right] \\
& M_{d r}=\left[\begin{array}{ccc}
0 & Q S / \operatorname{mass} V & 0 \\
a_{1} & \left(a_{1} / b\right)\left[\left(I_{x z} / I_{z z}\right) X_{f}-Z_{f}\right] & a_{1}\left(I_{x z} / I_{z z}\right) \\
a_{2} & \left(a_{2} / b\right)\left[X_{f}-\left(I_{x z} / I_{x x}\right) Z_{f}\right] & a_{2}\left(I_{x z} / I_{x x}\right)
\end{array}\right]
\end{aligned}
$$

Elements of the above matrices $a_{1}, a_{2}, A_{\dot{\beta} p}, A_{\dot{p}}, A_{\dot{r} p}, A_{\dot{\beta} r}, A_{\dot{p} r}$, and $A_{\dot{r} r}$ are defined in the Appendix. Vectors $V_{\beta}, V_{d a}$, and $V_{d r}$ are defined as

$$
V_{\beta}=\left[\begin{array}{l}
C l_{\beta} \\
C y_{\beta} \\
C n_{\beta}
\end{array}\right], \quad V_{d a}=\left[\begin{array}{l}
C l_{d a} \\
C y_{d a} \\
C n_{d a}
\end{array}\right], \quad V_{d r}=\left[\begin{array}{l}
C l_{d r} \\
C y_{d r} \\
C n_{d r}
\end{array}\right]
$$

Equation (10) for the lateral acceleration, $N_{y}$, can be rewritten in terms of $p, r, \dot{\beta}, \dot{p}$, and $\dot{r}$ :

$$
N_{y}=V[\dot{\beta}-\sin (\alpha) p+\cos (\alpha) r]+x_{a} \dot{r}-z_{a} \dot{p}
$$

The lateral-directional state-space Eqs. (3), (15), and (20) can be described as a block diagram in Fig. 1. Matrices $M_{p}$ and $M_{r}$ in the block diagram in Fig. 1 are

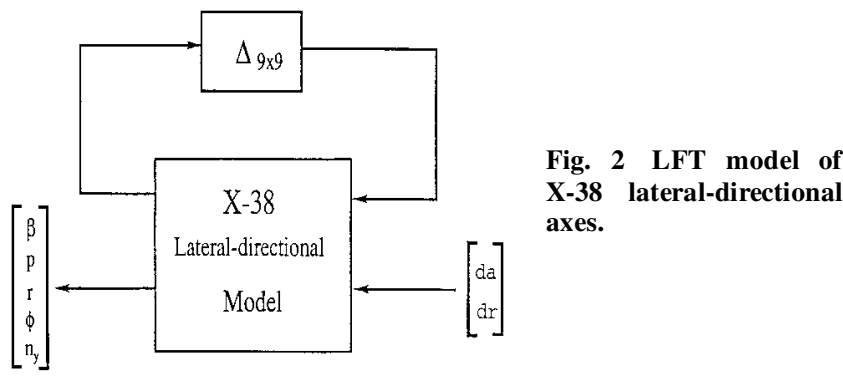

$$
\begin{gathered}
M_{p}^{T}=\left[\begin{array}{lllll}
A_{\dot{\beta} p} & A_{\dot{p} p} & A_{\dot{r} p} & 1 & -\sin (\alpha)
\end{array}\right] \\
M_{r}^{T}=\left[\begin{array}{lllll}
A_{\dot{\beta} r} & A_{\dot{p} r} & A_{\dot{r} r} & \tan (\theta) & \cos (\alpha)
\end{array}\right]
\end{gathered}
$$

From the block diagram, it is easy to isolate the unknown variables, the nine uncertainty parameters $\delta_{C i j}$, from the known data to construct an LFT model of the X-38 airframe.

Adding $\beta, p, r$, and $\phi$ outputs in the block diagram in Fig. 1, we can construct an X-38 LFT model with structured parameter uncertainties. The block diagram in Fig. 2 represents a generic framework for analyzing the system robustness. The $\Delta$ block has nine uncertainty real parameters as

$$
\begin{aligned}
& \Delta_{9 \times 9} \\
& \quad=\operatorname{diag}\left[\delta_{C l_{\beta}}, \delta_{C l_{d a}}, \delta_{C l_{d r}}, \delta_{C y_{\beta}}, \delta_{C y_{d a}}, \delta_{C y_{d r}}, \delta_{C n_{\beta}}, \delta_{C n_{d a}}, \delta_{C n_{d r}}\right]
\end{aligned}
$$

whose values range from -1 to 1 . The set of uncertainties cover the five $\sigma$ statistical variations of aerodynamic nondimensional stability derivatives. ${ }^{19}$ Each uncertain real parameter is independent of another, except the two parameters $\delta_{C l_{d r}}$ and $\delta_{C n_{d r}}$. We discuss how to handle two coupled uncertainty parameters in Sec. III.

\section{X-38 Analysis Interconnection Structure}

The objective of this paper is to analyze the robustness of the closed-loop lateral-directional axis to the uncertain aerodynamic 


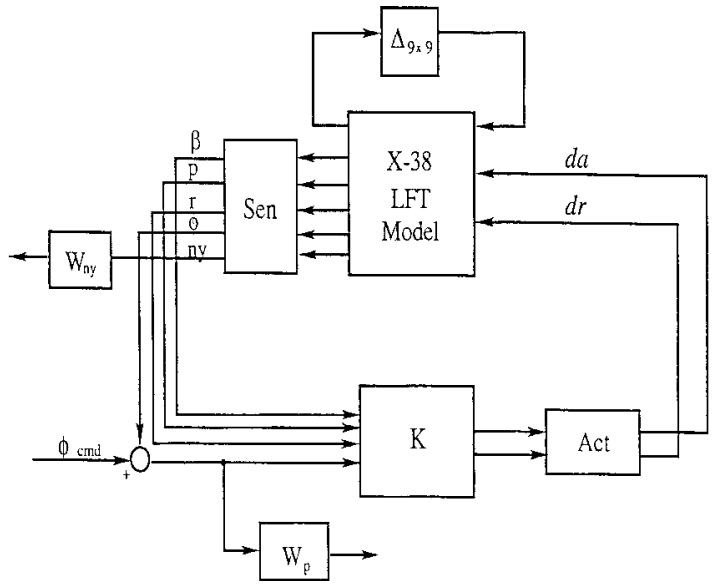

Fig. 3 Analysis interconnection of the X-38 lateral-directional axes.

coefficients. The closed-loop system for the X-38 lateral-directional axis can be constructed as shown in Fig. 3.

The performance objective for the lateral-directionalaxes is good low-frequency tracking of $\phi$ commands with the lateral acceleration kept small. These performance objectives are characterized in the analysis problem as $\mathcal{H}_{\infty}$ norm minimization of weighted transfer functions. The $\phi$ tracking objective is formulated as the weighted transfer function from the $\phi_{\text {cmd }}$ to $\phi_{\text {err }}$, with the objective to keep the weighted $\mathcal{H}_{\infty}$ norm below 1 . The weight $W_{p}$ is a first-order filter of the form $4[(s / 10+1) /(s+1)]$. This weight implies at low frequency, below $1 \mathrm{rad} / \mathrm{s}$, the closed loop should have less than $25 \%$ tracking error, which can degrade up to an error of $250 \%$ at high frequency. The lateral acceleration limit is included in the analysis problem formulation as a minimization of the $N_{y}$ response that is due to $\phi$ command inputs. A constant weight, $W_{N_{y}}$, of $\frac{1}{20}$ is used on the $N_{y}$ acceleration measurement. Therefore, for $\phi$ commands up to $1 \mathrm{rad}$, the lateral acceleration, $N_{y}$, should be smaller than $\frac{1}{20} g$. Models of the X-38 sensors and actuators are also included in the analysis problem formulation.

The X-38 has angle rate gyros modeled as a first-order system, $66 /(s+66)$, for roll rate $(p)$, yaw rate $(r)$, sideslip angle $(\beta)$, and roll angle $(\phi)$. The $N_{y}$ accelerometer is modeled as a first-order filter. The sensor model matrix, Sen, in Fig. 3 is

$$
\operatorname{Sen}=\left[\begin{array}{cc}
{[66 /(s+66)] I_{4 \times 4}} & 0 \\
0 & 40 /(s+40)
\end{array}\right]
$$

The X-38 EMAs are modeled as second-order systems, $26^{2} /\left(s^{2}+\right.$ $36.8 s+26^{2}$ ), which have a 0.707 damping ratio and less than $10 \%$ overshooting. Each EMA has a prefilter modeled as a second-order system $2500 /\left(s^{2}+70.7 s+2500\right)$. The actuator prefilter smoothes the prior discrete zero-order-hold output of a flight computer command output from a digital-to-analog converter to send it to the EMAs. ${ }^{19}$ There is also a transport delay modeled as a first Pade delay $(50-s) /(50+s)$. The Act block in Fig. 3 includes the two actuator models, prefilters, and transport delays:

$$
\text { Act }=\frac{50^{2}}{s^{2}+70.7 s+50^{2}} \frac{26^{2}}{s^{2}+36.8 s+26^{2}} \frac{50-s}{50+s} I_{2 \times 2}
$$

Consider the $\Delta_{9 \times 9}$ block, which includes the coupled real uncertainty parameters, $\delta_{C l_{d r}}$ and $\delta_{C n_{d r}}$. The correlation between uncertainty parameters of $\delta_{C l_{d r}}$ and $\delta_{C n_{d r}}$ is shown in Fig. 4 . The shaded region within the unit square in Fig. 4 (left) is the valid region. For simplicity of finding a mapping function, we assume that the valid region described in Fig. 4 represents uncertainties of $\delta_{C l_{d r}}$ and $\delta_{C n_{d r}}$. We define new uncertainty parameters such as $\delta_{C l_{d r}^{\prime}}$ and $\delta_{C n_{d r}^{\prime}}$ shown in Fig. 4 to be independent of each other. The mapping function from the independent parameters $\delta_{C l_{d r}^{\prime}}$ and $\delta_{C n_{d r}^{\prime}}$ to $\delta_{C l_{d r}}$ and $\delta_{C n_{d r}}$ is

$$
\begin{gathered}
\delta_{C l_{d r}}=\cos \left(45^{\circ}\right) \delta_{C l_{d r}^{\prime}}+\sin \left(45^{\circ}\right) \delta_{C n_{d r}^{\prime}} \\
\delta_{C n_{d r}}=-\sin \left(45^{\circ}\right) \delta_{C l_{d r}^{\prime}}+\cos \left(45^{\circ}\right) \delta_{C n_{d r}^{\prime}}
\end{gathered}
$$
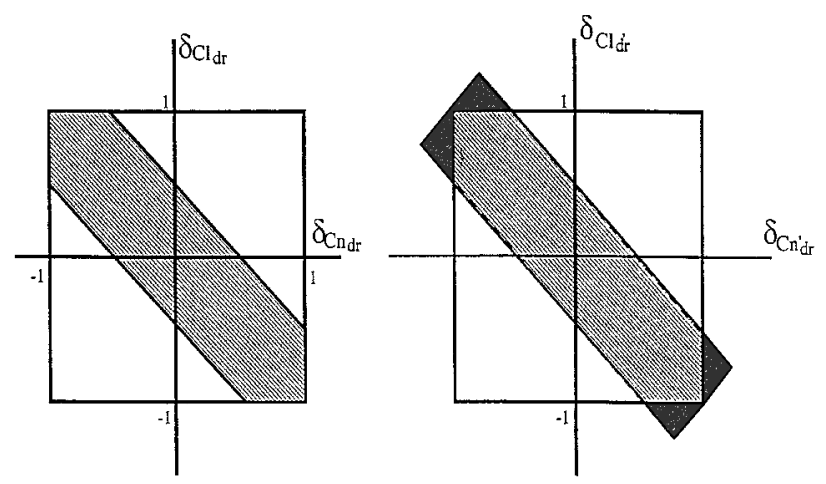

: Extra region in analysis

Fig. 4 Correlation of uncertainties of $C l_{d r}$ and $C n_{d r}$. (The dark area represents valid correlations.)
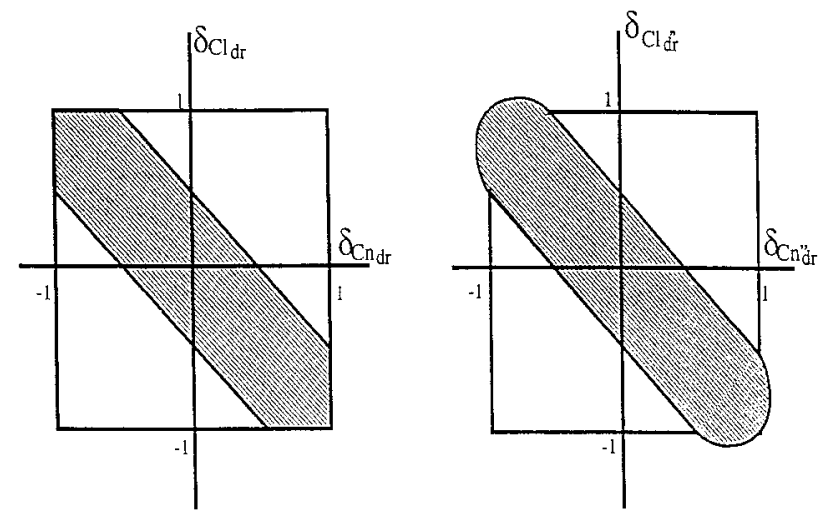

Fig. 5 The correlation of uncertainties of $C l_{\mathrm{dr}}$ and $C n_{\mathrm{dr}}$. (The dark area represents valid correlations.)

The parameter, $\delta_{C l_{d r}^{\prime}}$, varies from $-1 / 2 \sqrt{ } 2$ to $+1 / 2 \sqrt{ } 2$ and the parameter, $\delta_{C n_{d r}^{\prime}}$, varies from $-\sqrt{ } 2$ to $+\sqrt{ } 2$. This approximation allows $\delta_{C l_{d r}}$ to be 1.25 when $\delta_{C n_{d r}}$ is 0.75 in the extra region in Fig. 4 Using Eqs. (26), two copies of $\delta_{C l_{d r}^{\prime}}$ and $\delta_{C n_{d r}^{\prime}}$ can represent $\delta_{C l_{d r}}$ and $\delta_{C n_{d r}}$. The $\Delta$ block using new uncertainty parameters, $\delta_{C l_{d r}^{\prime}}$ and $\delta_{C n_{d r}^{\prime}}$, can be written as follows:

$$
\begin{gathered}
\Delta_{11 \times 11}=\operatorname{diag}\left[\delta_{C l_{\beta}}, \delta_{C l_{d a}}, \delta_{C y_{\beta}}, \delta_{C y_{d a}}, \delta_{C y_{d r}}, \delta_{C n_{\beta}}, \delta_{C n_{d a}},\right. \\
\left.\delta_{C l_{d r}^{\prime}} I_{2 \times 2}, \delta_{C n_{d r}^{\prime}} I_{2 \times 2}\right]
\end{gathered}
$$

The worst-case perturbation set based on the worst-case performance is found in the new $\Delta$ block. When values of $\delta_{C l_{d r}^{\prime}}$ and $\delta_{C n_{d r}^{\prime}}$, are found, the original values of $\delta_{C l_{d r}}$ and $\delta_{C n_{d r}}$ can be calculated from Eq. (26). Hereafter, method I correspond sto calculatingthe uncertain real parameters, $\delta_{C l_{d r}}$ and $\delta_{C n_{d r}}$, using the mapping function of Eq. (26).

A second approach, method II, uses a higher-ordermapping function from independentparameters, $\delta_{C l_{d r}^{\prime \prime}}$ and $\delta_{C n_{d r}^{\prime \prime}}$, to $\delta_{C l_{d r}}$ and $\delta_{C n_{d r}}$ to define more precisely the uncertainty in Fig. 4. The mapping function is written as

$$
\begin{aligned}
& \delta_{C l_{d r}}=0.25 \delta_{C l_{d r}^{\prime \prime}}-0.28 \delta_{C l_{d r}^{\prime \prime}}^{2} \delta_{C n_{d r}^{\prime \prime}}+\delta_{C n_{d r}^{\prime \prime}} \\
& \delta_{C n_{d r}}=0.25 \delta_{C l_{d r}^{\prime \prime}}+0.28 \delta_{C l_{d r}^{\prime \prime}}^{2} \delta_{C n_{d r}^{\prime \prime}}-\delta_{C n_{d r}^{\prime \prime}}
\end{aligned}
$$

where parameters $\delta_{C n_{d r}^{\prime \prime}}$ and $\delta_{C l_{d r}^{\prime \prime}}$ vary from -1 to 1 . Six copies of $\delta_{C l_{d r}^{\prime \prime}}$ and four copies of $\delta_{C n_{d r}^{\prime \prime}}$ in Eq. (28) are used to represent the coupled parameters, $\delta_{C l_{d r}}$ and $\delta_{C n_{d r}}$ (Fig. 5).

The $\Delta$ block can be written as follows:

$$
\begin{gathered}
\Delta_{17 \times 17}=\operatorname{diag}\left[\delta_{C l_{\beta}}, \delta_{C l_{d a}}, \delta_{C y_{\beta}}, \delta_{C y_{d a}}, \delta_{C y_{d r}}, \delta_{C n_{\beta}}, \delta_{C n_{d a}},\right. \\
\left.\delta_{C l_{d r}^{\prime}} I_{6 \times 6}, \delta_{C n_{d r}^{\prime}} I_{4 \times 4}\right]
\end{gathered}
$$

When values of $\delta_{C n_{d r}^{\prime \prime}}$ and $\delta_{C l_{d r}^{\prime \prime}}$ are found, the original value of $\delta_{C l_{d r}}$ and $\delta_{C n_{d r}}$ can be calculated from Eq. (28). 


\section{Worst-Case Analysis Algorithms}

The worst-case performance calculation is a branch-and-bound computation, using lower and upper bounds for the problem

$$
\max _{\Delta \in \Delta, \bar{\sigma}(\Delta) \leq 1} \bar{\sigma}\left[F_{U}(M, \Delta)\right]
$$

where $M$ is a given complex matrix, and $\Delta$ is a diagonal augmentation of scalar real parameters (possibly repeated many times), and complex matrices. A more detailed description of the worst-case analysis algorithm can be found in Ref. 21 . This method is different than the method described in Ref. 15, but it is motivated by many of the results there. We have not compared the method to those described in Ref. 17, but some comments follow.

In the case where $\Delta:=\{\delta I: \delta \in \boldsymbol{R}\}$, that is, a single real, repeated parameter, the problem

$$
\max _{a \leq \delta \leq b} \bar{\sigma}\left[F_{U}(M, \delta I)\right]
$$

has a clean numerical solution, using the ideas of state-space $\mathcal{H}_{\infty}$ norm calculation ${ }^{22,23}$ The lower bound is based on this, and a modified power algorithm for the complex blocks. ${ }^{11}$ The algorithm is as follows:

1) Initialize $\Delta$ at an allowable value.

2) Pick a random cycling order for the real perturbations.

3) Cycle through the real perturbations, holding all other perturbations fixed, and solve for maximizing the single real parameter, using Hamiltonian methods. Update that current parameter value with the new maximizer.

4) Holding the real parameters fixed, apply the power method to compute an approximately maximizing complex perturbation matrix (this is not performed element by element, but is done on all complex block simultaneously).

5) Return to step 2, and repeat.

The WRA algorithm in Ref. 17 is similar, in that the real perturbations are handled completely separately from the complex perturbations. Our method for maximization over the reals (simplistic coordinate at a time) is very different.

The upper bound is a standard scaled $-\mu$ upper bound ${ }^{24}$ and is formulated as a linear matrix inequality in scaling matrices $D$ and $G$, which depend on the uncertainty structure.
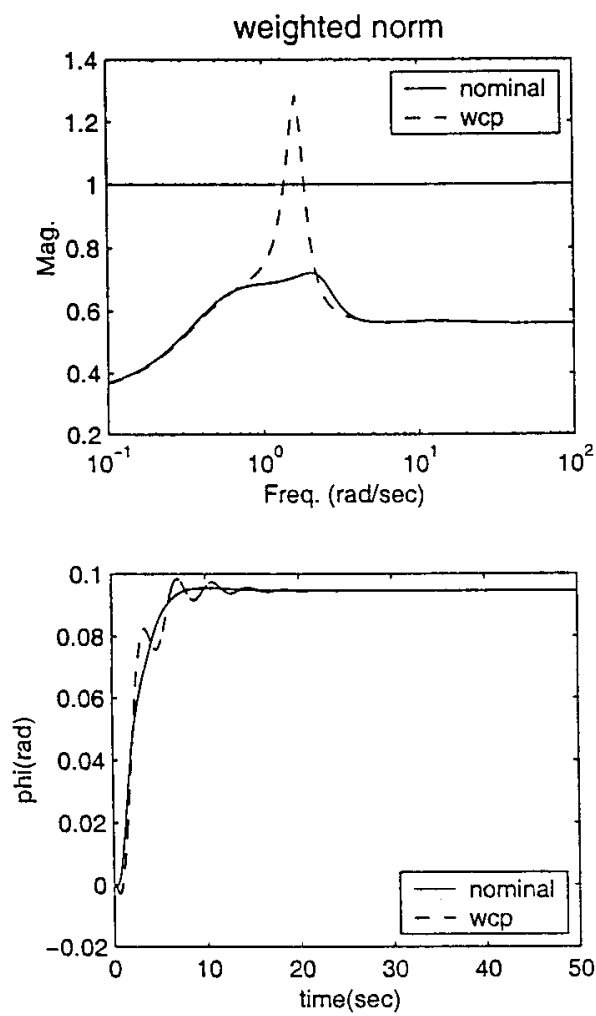

Finally, the bounds are improved by using a branch-and-bound computation on the real perturbations (but not the complex blocks). Because the upper and lower bounds are not guaranteed to converge for the complex blocks alone (except in special cases), the branchand-bounditeration is not guaranteed to convergeto the actual worstcase gain, but one should expect gaps similar to those seen in purely complex $\mu$ upper and lower bound calculations (which are typically small).

\section{Analysis Results}

The worst-case performance, robust stability, and robust performance of the X-38 closed-loopsystem is based on the block diagram shown in Fig. 3. There are two performance outputs, the tracking error $\phi_{\text {err }}$ and the weighted lateral-directional, $N_{y}$, and one exogenous input, roll angle command $\phi_{\text {cmd }}$. The weighted norm from $\phi_{\text {cmd }}$ to the two performance outputs should ne less than 1 for a nominal model, as the weighting function $W_{p}$ was derived based on nominal model response.

Consider the Mach 0.48, 29,000 ft altitude flight condition. The worst-case performance perturbations maximize the weighted norm at a single frequency point in the given frequency range, from 0.1 to $100 \mathrm{rad} / \mathrm{s}$. With the use of method I described in Sec. III, the worstcase performance delta set, $\Delta_{\mathrm{wc}_{I}}$, calculated at the flight condition is

$$
\Delta_{\mathrm{wc}_{I}}=\operatorname{diag}[1,-1,1.25,1,-1,1,-1,-1-0.75]
$$

where the order of uncertainty parameters in $\Delta$ is shown in Eq. (23). With the use of method II described in Sec. III,

$$
\Delta_{\mathrm{wc}_{I I}}=\operatorname{diag}[1,-1,1,1,-1,1,-1,-1,-0.77]
$$

When the calculated worst-case performance perturbations $\left(\Delta_{\mathrm{wc}_{I}}\right)$ are folded into the LFT model of the X-38 lateral-directional axes, the $\mathcal{H}_{\infty}$ norm from $\phi_{\text {cmd }}$ to the two performance outputs is 1.29 for the worst-case performance and 0.72 for the nominal case at the flight condition (Fig. 6). When $\Delta_{\text {wc }_{I I}}$ is used, the $\mathcal{H}_{\infty}$ norm is 1.23 for the worst-case performance. The defference between using $\Delta_{\mathrm{wc}_{I}}$ and $\Delta_{\mathrm{wc}_{I I}}$ is small enough to ignore it in the $\mathcal{H}_{\infty}$ norm respect. The analyais results shown in Fig. 6 are calculated by using $\Delta_{\mathrm{wc}_{I}}$. The maximum peak of the weighted norm occurs at the $1.7 \mathrm{rad} / \mathrm{s}$ frequency point for the worst-case performance. This implies that
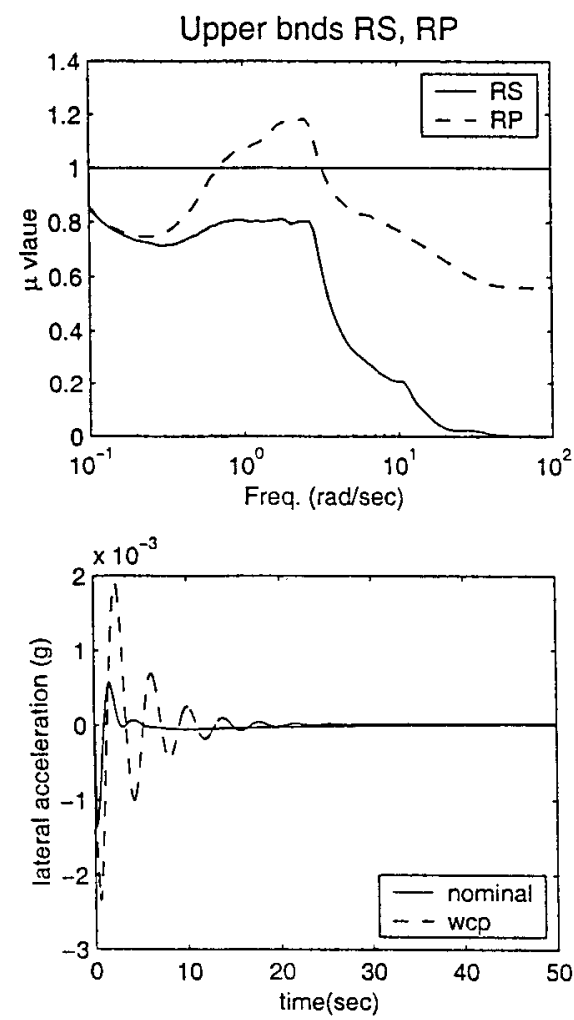

Fig. 6 Analysis results of the X-38 model at the 0.48-Mach, 29,000-ft-altitude flight condition: RS, robust stability; RP, robust performance. 
the $\phi$ and lateral acceleration step responses are oscillatory with a 3.7-s period (Fig. 6).

A linear simulation of $\phi$ and $N_{y}$ to a 0.1 -rad step input, $\phi_{\mathrm{cmd}}$, for nominal case and for worst-case performance perturbation is performed. The tracking error of $\phi$ is less than $6 \%$ at steady state for both nominal case and worst-case perturbations. The rising time for the $\phi$ step response is $8 \mathrm{~s}$. The change of lateral acceleration with a 0.1 -rad step roll angle command is $0.0024 \mathrm{~g}$, which slightly exceeds $( \pm 0.002 \mathrm{~g})$, the desired performance objective.

For robust stability of the controlled X-38 system, we calculated the structured singular value, $\mu$ of the X-38 system (Fig. 6) at the flight condition. It is difficult to calculate the lower bound of $\mu$ because of real parametric uncertainties of the aerodynamic coefficients for the X-38 system. ${ }^{2}$ It is sufficient to say that the conntrolled $\mathrm{X}-38$ system is stable because the $\mu$ robust stability upper bound is less than 1 (Fig. 6).

For robust performance of the X-38 system, we defined an augmented block structure $\Delta_{p}$ :

$$
\Delta_{p}=\left\{\left[\begin{array}{cc}
\Delta_{\text {unc }} & 0 \\
0 & \Delta_{f}
\end{array}\right]: \Delta_{\text {unc }} \in \Delta_{11 \times 11}, \Delta_{f} \in C^{1 \times 2}\right\}
$$

where $\Delta_{11 \times 11}$ set represents uncertain $\Delta$ structure described in Sec. III and $\Delta_{f}$ is a fictitious $\Delta_{f}$ set that is a full complex $1 \times 2$ matrix according to input and outputs of the system. The robust performance upper bound plot of $\mu$ is shown in Fig. 6. The maximum values of the lower and upper bounds of the $\mu$ value for robust performance in the frequency range $0.1-100 \mathrm{rad} / \mathrm{s}$ are 1.12 and 1.18 , respectively.

\section{Worst Gain and Phase Margins}

The X-38 V132 FCS has a requirement of a 4-dB gain margin and a 20-deg phase margin for all aerodynamiccoefficient variations within the set defined in Sec. II. Currently this requirementis verified at 128 test cases with predefined variations in the aerodynamic coefficients. Hence it is of interest to find the corresponding worst-case gain-and-phasemargin for each sensor and actuator channel for the entire range of aerodynamic coefficients. This involves a test of the aerodynamic uncertainty parameters that achieves the worst combined gain and phase margins. The combined analysis problem, to find the worst-case perturbation that minimizes the gain/phase margin, can be formulated with the addition of a feedback uncertainty block to individual actuator and sensor channels, respectively.

Figure 7 represents the block diagram for the combined margin analysis problem for the input aileron $(d a)$ channel. When the norm, $\left\|T F_{w_{1} \rightarrow z_{1}}\right\|_{\infty}$, is larger than 1 , the system is guaranteed to achieve a 6-dB gain and a 60-deg phase margin that can be calculated from a graphical interpretation of gain and phase margin in terms of the Nyquist locus plot. ${ }^{25,26}$ The $\mathcal{H}_{\infty}$ norm of $T F_{w_{1} \rightarrow z 1}$ defines the radius of the circle around the -1 point in the Nyquist plot as a function of frequency, which does not intersect with the loop gain of the system. The minimum gain margin is calculated from the intersection of the real axis and the norm circle centered at the -1 point. The minimum

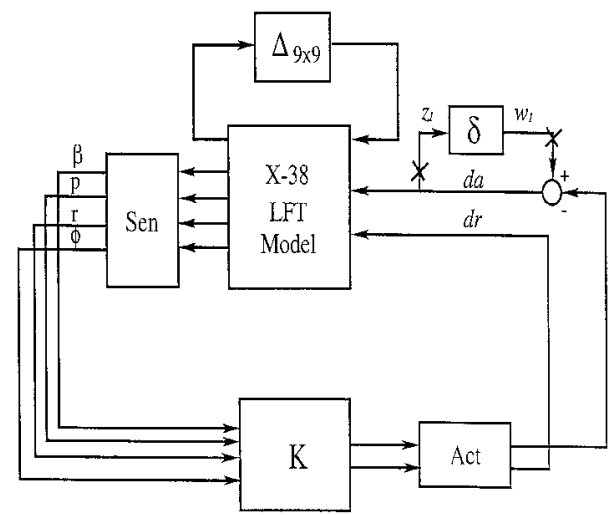

Fig. 7 The interconnection to calculate the gain and phase margins of the input $d a$ channel, using feedback uncertainty. Crosses indicate a $w_{1}$ input and $z_{1}$ output. phase margin is calculated from the intersection of the circle defined by the $\mathcal{H}_{\infty}$ norm of $T F_{w_{1} \rightarrow z_{1}}$ and the unit circle centered at the origin. From the graphicalinterpretationshown in Fig. 8 the minimum gain and phase margins can be written as follows:

$$
\begin{gathered}
G M_{\text {decrease }}=20 \log _{10}\left(C_{r}+1\right) \\
G M_{\text {increase }}=20 \log _{10}\left[1 /\left(1-C_{r}\right)\right] \\
P M=57.3 \sin ^{-1} \sqrt{\left[4-\left(C_{r}^{2}-2\right)^{2}\right] / 4}
\end{gathered}
$$

where

$$
C_{r}=1 /\left\|T F_{w_{1} \rightarrow z_{1}}\right\|_{\infty}
$$

With the use of Eqs. (33-35); the minimum gain and phase margin can be calculated in Table 1 from the calculated maximum $\left\|T F_{w_{1} \rightarrow z_{1}}\right\|_{\infty}$ for individual actuator and sensor channels, respectively.

The same method of calculating the worst-case performance $\Delta_{\mathrm{wc}}$ is used to calculate the worst-case combined margin perturbation $\Delta_{\text {wcgp }}$ :

$$
\Delta_{\text {wcgp }}=\left\{\Delta \in \Delta: \max \left\|T F_{w_{1} \rightarrow z_{1}}\right\|_{\infty}\right\}
$$

The worst-case margin perturbations $\Delta_{\mathrm{wcgp}}$ at the flight condition of 0.48 Mach and 29,000 ft altitude are shown in Table 2. The phase and gain margins in Table 3 are calculated by using the standard definition of gain and phase margin for each loop after the calculated

Table 1 Minimum gain and phase margins at the 0.48-Mach, 29,000-ft-altitude flight condition

\begin{tabular}{lcccc}
\hline \hline \multirow{2}{*}{$\begin{array}{l}\text { Loop } \\
\text { broken }\end{array}$} & \multirow{2}{*}{$\begin{array}{c}\text { Max } \\
F_{w_{1} \rightarrow z_{1}} \|_{\infty}\end{array}$} & Decrease & Increase & PM (deg) \\
\cline { 3 - 4 }$d a$ & 2.1 & 3.4 & 5.6 & \pm 28 \\
$d r$ & 2.0 & 3.5 & 5.9 & \pm 29 \\
$N_{y}$ & 1.2 & 5.2 & 14.6 & \pm 48 \\
$p$ & 2.1 & 3.3 & 5.5 & \pm 27 \\
$r$ & 2.0 & 3.5 & 6.0 & \pm 29 \\
$\phi$ & 1.4 & 4.8 & 11.6 & \pm 43 \\
\hline \hline
\end{tabular}

Table 2 Worst-case margin perturbation for each channel at the 0.48-Mach, 29,000-ft-altitude flight condition

\begin{tabular}{lllrrrrrrr}
\hline \hline Loop broken & & \multicolumn{10}{c}{$\Delta_{\text {wcgp }}$} \\
\hline$d a$ & $\operatorname{diag}[-1$ & -1 & 0.14 & -1 & -1 & 1 & -1 & 1 & $0.36]$ \\
$d r$ & $\operatorname{diag}[-1$ & -1 & 1.25 & 1 & -1 & 1 & -1 & -1 & $-0.75]$ \\
$N_{y}$ & $\operatorname{diag}[1$ & -1 & -0.75 & 1 & -1 & 1 & -1 & -1 & $1.25]$ \\
$p$ & $\operatorname{diag}[-1$ & -1 & 0.43 & -1 & -1 & 1 & -1 & 1 & $0.75]$ \\
$r$ & $\operatorname{diag}[1$ & 1 & 0.75 & 1 & 1 & -1 & 1 & 1 & $-1.25]$ \\
$\phi$ & $\operatorname{diag}[-1$ & -1 & -0.75 & 1 & -1 & 1 & -1 & -1 & $-1.25]$ \\
\hline \hline
\end{tabular}

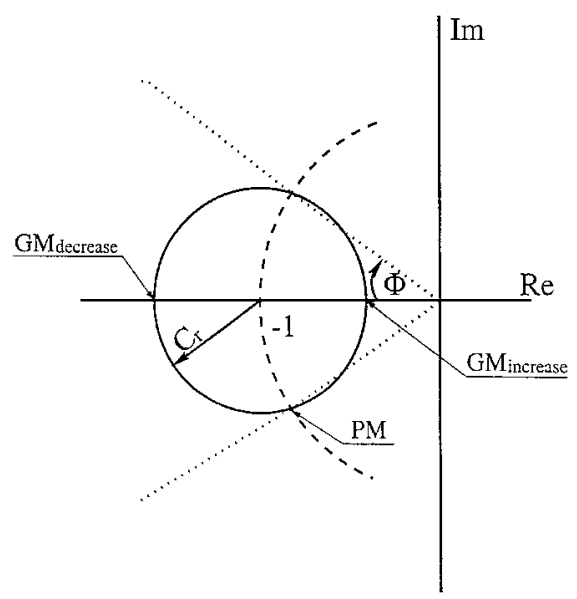

Fig. 8 Graphical interpretation of the gain and phase margin. $C_{r}$ represents the radius of a circle defined by $\left\|T F_{w_{1} \rightarrow z_{1}}\right\|_{\infty}$ at the point $(-1,0)$. 


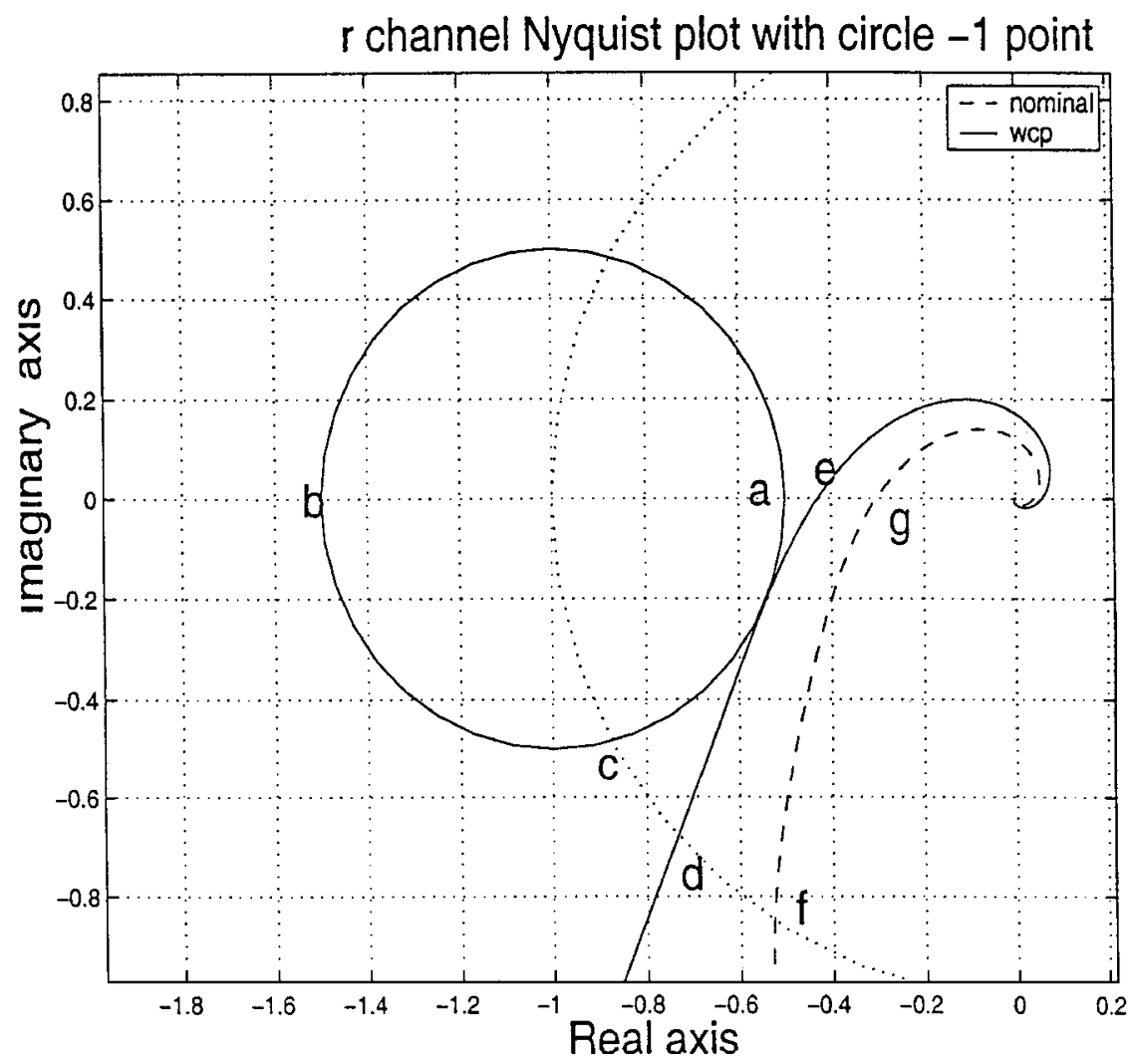

Fig. 9 Nyquist plots for the nominal case and the worst-case margins. Points a and $b$ represent gain increase margins and gain decrease margins in Table 1. Point $\mathrm{c}$ represents the phase margin in Table 1 . The worst gain and phase margins are represented by points e and $\mathrm{d}$. The gain and phase margins for the nominal case are represented by points $g$ and $f$.

Table 3 Worst-case gain and phase margins calculated at the 0.48-Mach, 29,000-ft-altitude flight condition ${ }^{\mathrm{a}}$

\begin{tabular}{lrccc}
\hline \hline $\begin{array}{l}\text { Loop } \\
\text { broken }\end{array}$ & $\begin{array}{c}\text { GM } \\
(\mathrm{dB})\end{array}$ & $\begin{array}{c}\text { Frequency } \\
(\mathrm{rad} / \mathrm{s})\end{array}$ & $\begin{array}{c}\text { PM } \\
(\mathrm{deg})\end{array}$ & $\begin{array}{c}\text { Frequency } \\
(\mathrm{rad} / \mathrm{s})\end{array}$ \\
\hline$d a$ & 20.4 & 10.7 & 28 & 2.8 \\
$d r$ & 8.9 & 11.1 & 31 & 1.8 \\
$N_{y}$ & 14.9 & 1.1 & 93 & 0.1 \\
$p$ & 25.2 & 11.2 & 29 & 2.7 \\
$r$ & 7.4 & 11.1 & 43 & 5.4 \\
$\phi$ & 11.9 & 0.7 & 83 & 0.1 \\
\hline \hline
\end{tabular}

${ }^{\mathrm{a}}$ When the worst-case margin perturbation for each channel is folded into the LFT model.

perturbations $\Delta_{\text {wcgp }}$ for each channel are folded into the X-38 LFT model. Comparing the gain and phase margins in Tables 1 and 3, one notices that the gain margins in Table 1 are similar to the gain margins in Table 3 for $N_{y}$ and $\phi$, respectively. For $d a, d r$, and $p$ channel, the phase margins in Table 1 are similar to those in Table 3 . The minimum gain and phase margin calculated in Table 1 from maximum $\left\|T F_{w_{1} \rightarrow z_{1}}\right\|_{\infty}$ captures the worst-case margins in Table 3. The Nyquist plots of nominal loop gain and the worst-case margin loop gain for the $r$ channel are shown in Fig. 9. From Fig. 9, it is obvious why the margins in Table 1 are smaller than the margins in Table 2. Calculation of the worst-case margin for all sensor and actuator channels at one flight condition takes approximately $80 \mathrm{~min}$ on a 300-MHz PII machine running Linux.

\section{Conclusions}

An X-38 LFT model of the linearized lateral-directional axis of the X-38 state-space equations is developed in this paper. This model is used to analyze the robustness of the closed-loopsystem to variations in the aerodynamic coefficients. Robust stability and robust performance of the X-38 lateral-directionalaxis are calculated. Worst-case performance and worst-case margins are calculated for the X-38 LFT model by bounding the levels of uncertainty to be of size 1 while maximizing the $\mathcal{H}_{\infty}$ norm of the performance transfer matrix.
The worst-case analysis results indicate that the closed-loop system achieves the 4-dB and 20-deg gain and phase margin requirements for all possible aerodynamic variations as defined by the aerodynamists at the 0.48 -Mach, 29,000-ft flight condition. The worstcase performance analysis indicates that there is only a small degradation in the performance of the X-38 lateral-directionalaxis in the presence of significant aerodynamic variations.

\section{Appendix: State-Space Equations}

The equations of the lateral-directionalmotion are

$$
\begin{aligned}
{\left[\begin{array}{c}
\dot{\beta} \\
\dot{p} \\
\dot{r} \\
\dot{\phi}
\end{array}\right] } & =\left[\begin{array}{cccc}
A_{\dot{\beta} \beta} & A_{\dot{\beta} p} & A_{\dot{\beta} r} & g \cos (\gamma) / V \\
A_{\dot{p} \beta} & A_{\dot{p} p} & A_{\dot{p} r} & 0 \\
A_{\dot{r} \beta} & A_{\dot{r} p} & A_{\dot{r} r} & 0 \\
0 & 1 & \tan (\theta) & 0
\end{array}\right]\left[\begin{array}{l}
\beta \\
p \\
r \\
\phi
\end{array}\right] \\
+ & {\left[\begin{array}{cc}
B_{\dot{\beta} d a} & B_{\dot{\beta} d r} \\
B_{\dot{p} d a} & B_{\dot{p} d r} \\
B_{\dot{r} d a} & B_{\dot{r} d r} \\
0 & 0
\end{array}\right]\left[\begin{array}{c}
d a \\
d r
\end{array}\right] }
\end{aligned}
$$$$
\left[\begin{array}{c}
\beta \\
p \\
r \\
\phi \\
N_{y}
\end{array}\right]=\left[\begin{array}{cccc}
1 & 0 & 0 & 0 \\
0 & 1 & 0 & 0 \\
0 & 0 & 1 & 0 \\
0 & 0 & 0 & 1 \\
C_{n_{y} \beta} & C_{n_{y} p} & C_{n_{y} r} & 0
\end{array}\right]\left[\begin{array}{c}
\beta \\
p \\
r \\
\phi
\end{array}\right]
$$$$
+\left[\begin{array}{cc}
0 & 0 \\
0 & 0 \\
0 & 0 \\
0 & 0 \\
D_{n_{y} d a} & D_{n_{y}} d r
\end{array}\right]\left[\begin{array}{c}
d a \\
d r
\end{array}\right]
$$

Each element of the state-space model is as follows: 


$$
\begin{aligned}
& A_{\dot{p} p}=\frac{a_{1}}{2 V}\left\{C l_{p}+\left(\frac{I_{x z} X_{f}}{I_{z z} b}-\frac{Z_{f}}{b}\right) C y_{p}\right. \\
& \left.+\frac{I_{x z}}{I_{z z}} C n_{p}+\frac{2 I_{p p} q_{0} V I_{x x}}{Q S b^{2}}\right\} \\
& A_{\dot{p} r}=\frac{a_{1}}{2 V}\left\{C l_{r}+\left(\frac{I_{x z} X_{f}}{I_{z z} b}-\frac{Z_{f}}{b}\right) C y_{r}\right. \\
& \left.+\frac{I_{x z}}{I_{z z}} C n_{r}+\frac{2 I_{p r} q_{0} V I_{x x}}{Q S b^{2}}\right\} \\
& A_{\dot{p} \beta}=a_{1}\left\{C l_{\beta}+\left(\frac{I_{x z} X_{f}}{I_{z z} b}-\frac{Z_{f}}{b}\right) C y_{\beta}+\frac{I_{x z}}{I_{z z}} C n_{\beta}\right\} \\
& A_{\dot{r} p}=\frac{a_{2}}{2 V}\left\{C n_{p}+\left(\frac{X_{f}}{b}-\frac{I_{x z} Z_{f}}{I_{x x} b}\right) C y_{p}\right. \\
& \left.+\frac{I_{x z}}{I_{x x}} C l_{p}+\frac{2 I_{r p} q_{0} V I_{z z}}{Q S b^{2}}\right\} \\
& A_{\dot{r} r}=\frac{a_{2}}{2 V}\left\{C n_{r}+\left(\frac{X_{f}}{b}-\frac{I_{x z} Z_{f}}{I_{x x} b}\right) C y_{r}\right. \\
& \left.+\frac{I_{x z}}{I_{x x}} C l_{r}+\frac{2 I_{r r} q_{0} V I_{z z}}{Q S b^{2}}\right\} \\
& A_{\dot{r} \beta}=a_{2}\left\{C n_{\beta}+\left(\frac{X_{f}}{b}-\frac{I_{x z} Z_{f}}{I_{x x} b}\right) C y_{\beta}+\frac{I_{x z}}{I_{x x}} C l_{\beta}\right\} \\
& A_{\dot{\beta} \beta}=\frac{Q S C y_{\beta}}{\operatorname{mass} V}, \quad A_{\dot{\beta} p}=\sin (\alpha)+\frac{Q S b C y_{p}}{2 \operatorname{mass} V^{2}} \\
& A_{\dot{\beta} r}=\frac{Q S b C y_{r}}{2 \operatorname{mass} V^{2}}-\cos (\alpha) \\
& B_{\dot{p} d a}=a_{1}\left\{C l_{d a}+\left(\frac{I_{x z} X_{f}}{I_{z z} b}-\frac{Z_{f}}{b}\right) C y_{d a}+\frac{I_{x z}}{I_{z z}} C n_{d a}\right\} \\
& B_{\dot{p} d r}=a_{1}\left\{C l_{d r}+\left(\frac{I_{x z} X_{f}}{I_{z z} b}-\frac{Z_{f}}{b}\right) C y_{d r}+\frac{I_{x z}}{I_{z z}} C n_{d r}\right\} \\
& B_{\dot{r} d a}=a_{2}\left\{C n_{d a}+\left(\frac{X_{f}}{b}-\frac{I_{x z} Z_{f}}{I_{x x} b}\right) C y_{d a}+\frac{I_{x z}}{I_{x x}} C l_{d a}\right\} \\
& B_{\dot{r} d r}=a_{2}\left\{C n_{d r}+\left(\frac{X_{f}}{b}-\frac{I_{x z} Z_{f}}{I_{x x} b}\right) C y_{d r}+\frac{I_{x z}}{I_{x x}} C l_{d r}\right\} \\
& B_{\dot{\beta} d a}=\frac{Q S C y_{d a}}{\operatorname{mass} V}, \quad B_{\dot{\beta} d r}=\frac{Q S C y_{d r}}{\operatorname{mass} V} \\
& C_{n_{y} \beta}=V A_{\dot{\beta} \beta}+X_{a} A_{\dot{r} \beta}-Z_{a} A_{\dot{p} \beta} \\
& C_{n_{y} p}=\frac{Q S b C y_{p}}{2 \operatorname{mass} V}+X_{a} A_{\dot{r} p}-Z_{a} A_{\dot{p} p} \\
& C_{n y r}=\frac{Q S b C y_{r}}{2 \operatorname{mass} V}+X_{a} A_{\dot{r} r}-Z_{a} A_{\dot{p} r} \\
& D_{n_{y} d a}=\frac{Q S C y_{d a}}{\operatorname{mass}}+X_{a} A_{\dot{r} d a}-Z_{a} A_{\dot{p} d a} \\
& D_{n_{y} d r}=\frac{Q S C y_{d r}}{\operatorname{mass}}+X_{a} A_{\dot{r} d r}-Z_{a} A_{\dot{p} d r} \\
& a_{1}=D_{I} \frac{Q S b^{2}}{I_{x x}}, \quad a_{2}=D_{I} \frac{Q S b^{2}}{I_{z z}}
\end{aligned}
$$

$$
\begin{array}{lc}
I_{p p}=\frac{I_{x z}}{I_{x x}}+\frac{I_{x z}\left(I_{x x}-I_{y y}\right)}{I_{x x} I_{z z}}, & I_{p r}=\frac{I_{y y}-I_{z z}}{I_{x x}}-\frac{I_{x z}^{2}}{I_{x x} I_{y y}} \\
I_{r p}=\frac{I_{x z}^{2}}{I_{x x} I_{z z}}+\frac{I_{x x}-I_{y y}}{I_{z z}}, & I_{r r}=\frac{I_{x z}\left(I_{y y}-I_{z z}\right)}{I_{z z} I_{x x}}-\frac{I_{x z}}{I_{y y}} \\
D_{I}=\frac{I_{x x} I_{z z}}{I_{x x} I_{z z}-I_{x z} I_{x z}} &
\end{array}
$$

\section{Acknowledgments}

This work was funded under Liberated Staffing Systems/ Lockheed Martin/NASA Johnson ContractLTD039. The Technical Monitor for this work was Steve Munday at NASA Johnson Space Center. The authors acknowledge the help of Dale Enns and Dan Bugjaski of the Honeywell Technology Center in developing the linearized lateral-directionalmodel of the X-38 and formulation of the control analysis problem.

\section{References}

${ }^{1}$ Zhou, K., Doyle, J., and Glover, K., Robust and Optimal Control, Prentice-Hall, Upper Saddle River, NJ, 1996, pp. 247-300.

${ }^{2}$ Young, P., Newlin, M., and Doyle, J., " $\mu$ Analysis with Real Parametric Uncertainty," Proceedings of the 30th IEEE Conference on Decision and Control, Inst. of Electrical and Electronics Engineers, New York, Vol. 2, 1991, pp. 1251-1256.

${ }^{3}$ Belcastro, C., "Parametric Uncertainty Modeling: An Overview," Proceedings of the American Control Conference, IEEE Publications, Piscataway, NJ, Vol. 2, 1998, pp. 992-996.

${ }^{4}$ Lambrechts, P., Terlouw, J., Bennai, S., and Steinbuch, M., "Parametric Uncertainty Modeling Using LFTs," Proceedings of the American Control Conference, IEEE Publications, Piscataway, NJ, Vol. 1, 1993, pp. 267272.

${ }^{5}$ Cockburn, J. C., "Linear Fractional Representations of Systems with Rational Uncertainty," Proceedings of the American Control Conference, IEEE Publications, Piscataway, NJ, Vol. 2, 1998, pp. 1008-1012.

${ }^{6}$ Ray, L. R., and Stengel, R. F., "Stochastic Measurement of Performance in Aircraft Control Systems," Journal of Guidance, Control, and Dynamics, Vol. 15, No. 6, 1992, pp. 1381-1387.

${ }^{7}$ Stengel, R. F., and Ray, L. R., "Stochastic Robustness of Linear Time-Invariant Control System," IEEE Transactions on Automatic Control, Vol. 36, No. 1, 1991, pp. 82-87.

${ }^{8}$ Morton, B., and McAfoos, R., "A Mu-Test for Robustness Analysis of Real-Parameter Variation Problem," Proceedings of the American Control Conference, IEEE Publications, Piscataway, NJ, Vol. 1, 1985, pp. 135138.

${ }^{9}$ Doyle, J., Lenz, J. K., and Packard, A., "Design Examples Using $\mu$ Synthesis: Space Shuttle Lateral Axis FCS During Reentry," Modelling, Robustness, and Sensitivity Reduction in Control Systems, NATO ASI Series, Vol. 34, Springer-Verlag, New York, 1987.

${ }^{10}$ Young, P., and Doyle, J., "Computation of $\mu$ with Real and Complex Uncertainties," Proceedings of the 29th IEEE Conference on Decision and Control, Inst. of Electrical and Electronics Engineers, New York, Vol. 2, 1990, pp. 1230-1235.

${ }^{11}$ Packard, A. K., "The Complex Structured Singular Value," Automatica, Vol. 29, No. 1, 1993, pp. 71-109.

${ }^{12}$ Young, P., Newlin, M., and Doyle, J., "Practical Computation of the Mixed $\mu$ Problem," Proceedings of the American Control Conference, IEEE Publications, Piscataway, NJ, 1992, pp. 2190-2194.

${ }^{13}$ Fan, M., and Tits, A., "Characterization and Efficient Computation of the Structured Singular Value," IEEE Transactions on Automatic Control, Vol. AC-31, No. 8, 1986, pp. 734-743.

${ }^{14}$ Newlin, M., and Glavaski, S., "Advances in the Computation of the $\mu$ Lower Bound," Proceedings of the American Control Conference, IEEE Publications, Piscataway, NJ, 1995, pp. 442-446.

${ }^{15}$ Newlin, M., and Young,P., "Mixed mu Problems and Branch and Bound Techniques," International Journal of Robust Nonlinear Control, Vol. 7 , No. 2, 1997, pp. 145-164.

${ }^{16}$ Balakrishnan, V., Boyd, S., and Balemi, S., "Branch and Bound Algorithm for Computing the Minimum Stability Degree of Parameter-Dependent Linear System," International Journal of Robust and Nonlinear Control, Vol. 1, No. 4, 1991, pp. 295-317.

${ }^{17}$ Glavaski, S., and Tierno, J., "Advances in Worst-Case $\mathcal{H}_{\infty}$ Performance Computation," Proceedings of the 1998 IEEE International Conference on Control Applications, Inst. of Electrical and Electronics Engineers, New York, 1998, pp. 668-673.

${ }^{18}$ Nelson, R. C., Flight Stability and Automatic Control, McGraw-Hill, New York, 1998, pp. 181-211, Chap. 5. 
${ }^{19}$ Ruppert, J. P., Munday, S., Estes, J., and Merkle, S., "X-38 Vehicle 132 Guidance, Navigation, and Control system," Design Workbook, NASA Johnson Space Center, Houston, TX, Nov. 1998.

${ }^{20}$ Balas, G., Doyle, J., Glover, K., Packard, A., and Smith, R., $\mu$ Analysis and Synthesis Toolbox, MathWorks, Natick, MA, 1995, pp. 47-81, Chap. 4.

${ }^{21}$ Packard, A., Balas, G., Liu, R., and Shin, J.-Y., "Results on Worst-Case Performance Assessment," Proceedings of the American Control Conference, IEEE Publications, Piscataway, NJ, 2000, pp. 2425-2427.

${ }^{22}$ Boyd, S., Balakrishnan, V., and Kabamba, P., "A Bisection Method for Computing the $\mathcal{H}_{\infty}$ Norm of a Transfer Matrix and Related Problems," Math Control Signals and Systems, Vol. 2, No. 3, 1989, pp. 207-219.
${ }^{23}$ Bruinsma, M., and Steinbuch, M., "A Fast Algorithm to Compute the $\mathcal{H}_{\infty}$ Norm of a Transfer Function Matrix," Systems and Control Letters, Vol. 14, No. 4, 1990, pp. 287-293.

${ }^{24}$ Fan, M., Tits, A., and Doyle, J., "Robustness in the Presence of Joint Parametric Uncertainty and Unmodeled Dynamics," IEEE Transactions on Automatic Control, Vol. 36, No. 1, 1991, pp. 25-38.

${ }^{25}$ Maciejowski, J. M., Multivariable Feedback Design, Addison Wesley Longman, Reading, MA, 1989, p. 227.

${ }^{26}$ Safonov, M., and Athans, M., "Gain and Phase Margin for Multiloop LQG Regulators," IEEE Transactions on Automatic Control, Vol. AC-22, No. 2, 1977, pp. 173-179. 